Published in final edited form as:

Ann Epidemiol. 2011 August ; 21(8): 580-588. doi:10.1016/j.annepidem.2011.03.004.

\title{
Variation in Rates of Fatal Coronary Heart Disease by Neighborhood Socioeconomic Status: The Atherosclerosis Risk in Communities Surveillance (1992-2002)
}

\author{
Randi E Foraker, $\mathrm{PhD}^{1}$, Kathryn M Rose, $\mathrm{PhD}^{2}$, Anna M Kucharska-Newton, $\mathrm{PhD}^{3}$, Hanyu \\ $\mathrm{Ni}, \mathrm{PhD}^{4}$, Chirayath M Suchindran, $\mathbf{P h D}^{5}$, and Eric A Whitsel, MD, MPH ${ }^{3,6}$ \\ ${ }^{1}$ Division of Epidemiology, College of Public Health, The Ohio State University, Columbus, Ohio, \\ USA \\ 2SRA International, Durham, North Carolina, USA \\ ${ }^{3}$ Department of Epidemiology, Gillings School of Global Public Health, University of North \\ Carolina, Chapel Hill, North Carolina, USA \\ ${ }^{4}$ Department of National Heart, Lung and Blood Institute, National Institutes of Health, Bethesda, \\ Maryland, USA \\ ${ }^{5}$ Department of Biostatistics, Gillings School of Global Public Health, University of North Carolina, \\ Chapel Hill, North Carolina, USA \\ ${ }^{6}$ Department of Medicine, Gillings School of Global Public Health, University of North Carolina, \\ Chapel Hill, North Carolina, USA
}

\section{Abstract}

Purpose-Racial and gender disparities in out-of-hospital deaths from coronary heart disease (CHD) have been well-documented, yet disparities by neighborhood socioeconomic status have been less systematically studied in US population-based surveillance efforts.

\begin{abstract}
Methods-We examined the association of neighborhood socioeconomic status (nSES), classified into tertiles, with 3,743 out-of-hospital fatal CHD events, and a subset of 2,191 events classified as sudden, among persons aged 35 to 74 years in four US communities under surveillance by the Atherosclerosis Risk in Communities (ARIC). Poisson generalized linear mixed models generated age-, race- (white, black) and gender-specific standardized mortality rate ratios and $95 \%$ confidence intervals ( $\mathrm{RR}, 95 \% \mathrm{CI}$ ).
\end{abstract}

Results-Regardless of nSES measure used, inverse associations of nSES with all out-ofhospital fatal CHD and sudden fatal CHD were seen in all race-gender groups. The magnitude of these associations was larger among women than men. Further, among blacks, associations of low nSES (vs. high nSES) were stronger for sudden deaths than for all out-of-hospital fatal CHD.

\footnotetext{
(C) 2011 Elsevier Inc. All rights reserved.

Corresponding Author / Reprint Request: Randi E. Foraker, Division of Epidemiology, The Ohio State University, $320 \mathrm{~W} .10^{\text {th }}$ Avenue, Columbus, OH 43210, 614-293-3919 (Tel), 614-293-3937 (Fax), rforaker@cph.osu.edu.

Publisher's Disclaimer: This is a PDF file of an unedited manuscript that has been accepted for publication. As a service to our customers we are providing this early version of the manuscript. The manuscript will undergo copyediting, typesetting, and review of the resulting proof before it is published in its final citable form. Please note that during the production process errors may be discovered which could affect the content, and all legal disclaimers that apply to the journal pertain.
} 
Conclusions-Low nSES was associated with an increased risk of out-of-hospital CHD death and SCD. Measures of the neighborhood context are useful tools in population-based surveillance efforts for documenting and monitoring socioeconomic disparities in mortality over time.

\section{Keywords}

Out-of-hospital death; surveillance; socioeconomic status

\section{INTRODUCTION}

Coronary heart disease (CHD) mortality has declined in recent decades (1-8). The majority of CHD deaths are out-of-hospital $(1,7)$, defined as deaths due to cardiac causes occurring outside of a hospital or upon arrival at the emergency room. Sudden cardiac death (SCD) occurs within one hour of the onset of symptoms. Extant literature suggests that half of all CHD deaths are sudden $(9,10)$, and that the majority of SCD cases occur out-of-hospital (11).

Declines in CHD mortality are more pronounced in men as compared to women (1-3, 5-8). Racial disparities in out-of-hospital cardiac arrest also exist, as incidence appears higher and survival lower among blacks compared to whites (11). A report based on Atherosclerosis Risk in Communities (ARIC) surveillance data from 1987 through 1994 suggests that annual declines in CHD mortality were steepest among white men (4.7\%), followed by white women $(4.5 \%)$, black women $(4.1 \%)$ and black men $(2.5 \%)$, respectively (6). These data also indicate that among women, approximately half of CHD deaths occurred out-ofhospital, while for men, $64 \%$ of CHD deaths were out-of-hospital (6). ARIC community surveillance data (1987-2004) suggest that SCD has declined with time, yet trends differ by community and gender (12).

Out-of-hospital death and SCD display geographic variation (12-14) which may be at least partly due to factors associated with neighborhood socioeconomic status (nSES). In the United States (US), the influence of nSES on rates of fatal out-of-hospital CHD has not been systematically examined. In this study we use data from the ARIC community surveillance study to examine the association of nSES and the risk of out-of-hospital CHD death, also referred to herein as out-of-hospital fatal CHD, and SCD as well as the utility of incorporating nSES in population-based surveillance efforts.

\section{METHODS}

The Atherosclerosis Risk in Communities (ARIC) study's community surveillance began in 1987 to evaluate trends in CHD morbidity and mortality in four US communities: Forsyth County, North Carolina (NC); the city of Jackson, Mississippi (MS); suburbs of Minneapolis, Minnesota (MN); and Washington County, Maryland (MD). Detailed methods for ascertaining and classifying fatal events for surveillance investigation are described elsewhere (15). Briefly, death certificates for ARIC community residents meeting age and cause of death criteria were sampled based on underlying cause of death International Classification of Diseases (ICD), Version 9 codes and were subsequently reviewed by trained abstractors. Sampling weights, based upon the sampling fractions, were used in subsequent analyses.

Two physicians from the ARIC Mortality and Morbidity Classification Committee independently assigned a cause of death and applied a CHD death classification based on data collected regarding chest pain, medical history, and other probable causes of death and underlying cause of death (ICD) codes. As a result, deaths were classified into one of the 
following five categories: 1) Definite fatal myocardial infarction, requiring evidence of a hospitalized definite myocardial infarction during the previous 28 days; 2) Definite fatal CHD; 3) Possible fatal CHD; 4) Non-CHD death; or 5) Unclassifiable. Definite and possible CHD deaths were further classified as sudden if they occurred within one hour from the onset of symptoms. Unknown timing of death precluded assignment of a sudden death classification.

Out-of-hospital deaths were defined as those occurring outside of the hospital, among community members in nursing homes, or persons declared dead on arrival, who died in outpatient departments or emergency rooms, as well as those admitted without vital signs (16). For deaths occurring out-of-hospital, next of kin, family physicians and medical examiners were contacted to complete information regarding timing of death, symptoms experienced prior to death, and medical history.

Decedents' addresses were obtained from death certificates and geocoded to the level of the census tract (CT) by a vendor with known accuracy and repeatability(17). Year 2000 CTlevel nSES was assigned based on decedent place of residence. The single-variable CT-level measures selected for study included: median household income (nINC), percent of persons below poverty, percent female-headed households, percent college-educated and percent high school-educated. A composite CT-level index, as used by Diez-Roux and Borrell, was comprised of six indicators of income/wealth $(18,19)$. We classified nSES variables into tertiles of low (L-), medium (M-) and high (H-). For additional comparison purposes, tertiles were calculated based on nSES: 1) overall; across all ARIC study communities, 2)

community-specific; within each study community, and 3) race-specific; within race groups (Table 1). We used CT-level measures to define the neighborhood context. This approach has been previously used by our group (20), as well as other researchers (19-21). Decennial census-based CT-level socioeconomic characteristics are standardized and available for the entire US; further, CTs are designed to be sociodemographically homogenous units (22).

Additional variables considered in the subsequent analyses, when applicable, included: race (black or white), gender, age (eight strata: 35-39; 40-44; 45-49; 50-54; 55-59; 60-64; 65-69; 70-74), study community and year of death (1992-1995, 1996-1999, 2000-2002). For the current study, ARIC community surveillance data were analyzed over the time period 1992-2002, as address data were not available prior to 1992. Figure 1 shows exclusions for the current study. Deaths were excluded if missing information on CHD classification, death dates were outside the date range specified for study, or if records were missing key demographic or sociodemographic information. Out of an initial 4,868 out-of-hospital deaths, 3,743 (4,336 weighted) were available for the analysis, and a subset of 2,191 (2,559 weighted) were identified as eligible sudden fatal CHD.

For the analysis of out-of-hospital and SCD, tract-level population counts from the 1990 and 2000 US Census were normalized to the CT boundaries from the 2000 census (23). We used a simple linear interpolation/extrapolation method to calculate age-, gender-, and racespecific population estimates for inter-censual and post-censual periods for each CT. The total year 2000 population distribution for all ARIC study sites, summed across age, gender and race, was used as the standard population. A weighted mortality count for years 1999-2001 was averaged within eight age strata in order to produce age-specific death rates for the standard population. We calculated indirect standardized rates for each tract by applying the age-specific death rates from the standard population to the tract-level population counts, yielding the expected number of (age-, gender-, and race-specific) deaths for each CT. 
We used the number of expected deaths and the number of observed deaths, weighted according to sampling criteria, to calculate standardized mortality ratios (SMR). We estimated out-of-hospital mortality rate ratios (RR) using Poisson generalized linear mixed model regression (PROC GLIMMIX, SAS 9.1, Cary, NC). In order to examine if nSES effects varied across time, the Poisson models were further extended to include both time (year of death) and time interactions. A subset of analyses using the methods described above was also conducted for out-of-hospital SCD. We additionally identified factors associated with unknown timing of death and conducted a sensitivity analysis of SCD, applying weights to each race-gender group based on their predicted probability of having an unknown timing of death. We assessed covariates as potential effect modifiers of the nSES-mortality relationship $(\mathrm{p}<0.05)$.

\section{RESULTS}

Year 2000 US Census characteristics of the eligible study population from which out-ofhospital deaths were assessed for this study are described in Table 2 . Whites comprised the majority of the MD and MN populations, while blacks were more highly represented MS and NC. The average nINC across all four communities ranged from $\$ 25,480$ in MS to $\$ 54,508$ in $\mathrm{MN}$.

Of all eligible out-of-hospital fatal CHD $(\mathrm{N}=4,336)$ and $\mathrm{SCD}(\mathrm{N}=2,559)$ events, white men comprised the highest number in both categories: 2,184 (50\%) and 1,303 (51\%), respectively. Black women had the fewest fatal CHD $(n=489,11 \%)$ and SCD $(n=291,11 \%)$ events of all race-gender groups, while white women [862 (20\%) and $459(18 \%)$, respectively] and black men [801 (19\%) and 506 (20\%), respectively] had the next highest number of fatal CHD and SCD events.

\section{Median household income (nINC)}

Regardless of nINC cutpoints used, white men had the highest absolute age-adjusted rates of out-of-hospital fatal CHD per 100,000 persons in the ARIC study communities, followed by black men, black women and white women (Figure 2). Meanwhile, residents of MS experienced the highest rates of out-of-hospital CHD death, and persons living MN recorded the lowest rates for the time period 1992-2002 (Figure 2).

Looking across study community and race-gender group, respectively, persons living in LnINC areas at the time of their death had higher rates of out-of-hospital fatal CHD than those living in H-nINC areas (Figure 2). With the exception of black men, rates of out-ofhospital CHD death were also higher for persons from M-nINC areas than rates observed in $\mathrm{H}$-nINC areas. These nINC-mortality gradients persisted regardless of nINC cutpoints used. While similar associations held in the investigation of out-of-hospital SCD, differences between race-gender groups and study communities, respectively, were not as pronounced, due to decreased precision caused by a smaller number of events (data not shown).

Race-gender differences were observed for all out-of-hospital deaths. Neither study community nor time (year of death) significantly modified the nINC-mortality relationship. Thus, Tables 3 and 4 present RR [95\% confidence interval (CI)] for out-of-hospital CHD deaths by race-gender group, adjusted for study community and year of death. Inverse associations of nINC with all out-of-hospital fatal CHD (Table 3) and sudden fatal CHD events (Table 4) were seen in all race-gender groups. The magnitude of these associations was larger among women than men. Further, among blacks, associations of low nINC (vs. high nINC) were stronger for sudden deaths than for all out-of-hospital fatal CHD. 


\section{Other measures of nSES}

Patterns similar to those described for the nINC-mortality relationship (Tables 3 and 4) held for all other measures of nSES (see Supplementary Figure 1). The sensitivity analysis of $\mathrm{SCD}$, which employed weights based upon the predicted probability of having an unknown time of death, produced results similar to those in Table 4 for each measure of nSES (data not shown).

Although inverse associations between nSES and all out-of-hospital fatal CHD and sudden fatal CHD were seen in all race-gender groups, the magnitude of these associations was generally stronger among women than men. We observed increasing rates of out-of-hospital death (Table 3) and SCD (Table 4) with lower nSES for both whites and blacks. For out-ofhospital death and SCD, whites living in L-nSES and M-nSES areas prior to death had an elevated risk of out-of-hospital death, but living in a L-nSES area carried a greater risk than living in a M-nSES area compared to those living in H-nSES areas. Meanwhile, blacks living in L-nSES areas tended to have an elevated risk of out-of-hospital fatal CHD (Table 3) and SCD (Table 4) compared to blacks living in H-nSES areas, while blacks living in MnSES areas did not.

\section{DISCUSSION}

Although race and gender disparities in out-of-hospital deaths have been previously documented $(5,11)$, differences in rates of out-of-hospital fatal CHD by neighborhood socioeconomic factors have not been systematically studied. Since address data are readily available on death certificates, and decedents' addresses can be geocoded to determine place of residence at the time of death, CHD surveillance efforts can be expanded to incorporate measures of the neighborhood context in order to examine socioeconomic disparities in mortality over time.

Across all race-gender groups and study communities, out-of-hospital death rates in ARIC community surveillance were generally highest among residents of L-nSES areas and lowest among persons living in H-nSES areas at the time of death. The magnitude of the nSESmortality associations tended to be larger among women than men. We observed an inverse nSES-mortality relationship across all levels of nSES among whites, but a significant inverse relationship only existed between L-nSES and H-nSES among blacks.

In these data, the nSES-mortality relationships and their interpretations were similar, regardless of whether the nSES measure was 1) Classified using overall, communityspecific or race-specific cutpoints; 2) Defined by income, poverty, female headship rates or educational attainment; or 3) A single-variable as compared to a composite-variable. Comparable findings were described by Krieger et al. in an investigation of the relation of area-based SES measures to a variety of health outcomes (24). Our results were also consistent with a study of 19 countries, which concluded that socioeconomic inequalities in malnutrition were of similar magnitude regardless of choice of SES measure (25).

Although the magnitude and direction of our nSES-mortality estimates did not vary according to which nSES measure was used, we acknowledge that there is often a theoretical basis for the choice of nSES measure (26). Selection of nSES measures for study are frequently based upon a priori hypotheses regarding the relevance of a particular measure to a population, or efforts to understand the mechanism by which the chosen area-based measure of SES influences health. Investigators utilizing composite measures of nSES should keep in mind the population for which the nSES index was developed, and determine whether it is appropriate for use in a distinct study setting. The composite nSES measure chosen for this analysis was developed using factor analysis of data from the ARIC study 
cohort, thus, the composite measure is likely appropriate for the ARIC community surveillance population.

The ongoing community surveillance and classification of deaths in ARIC is a strength of the current study. The ability to link death certificate data with ARIC surveillance hospitalization records, which provide information on the occurrence of a definite or probable MI within 28 days of death, strengthens the definition of CHD-related deaths. It should be acknowledged, however, that not all sources of data - hospitalization records, coroner reports, and next-of-kin or witness interviews - are available for all decedents identified in ARIC surveillance. The classification of death is more accurate when complete data are available on the timing of death, eyewitness reports and recent physician visits.

In particular, unknown timing of death in relation to symptom onset precludes the classification of SCD. In our study, $17 \%$ of persons otherwise meeting out-of-hospital fatal CHD criteria were missing information on time elapsed since symptom onset. Deaths that are not witnessed cannot be included in an analysis of SCD, even though it is estimated that half of all out-of-hospital deaths are due to SCD. We classified nearly $60 \%$ of out-ofhospital fatal CHD as sudden. However, it is possible that the number of SCDs were underestimated in this study due to missing data on the timing of death.

In our study, decedents missing data on the timing of death but otherwise meeting out-ofhospital fatal CHD criteria were more likely to be male $(66.4 \%)$, white $(63.4 \%)$ and residing in L-nINC (49.6\%) or M-nINC (33.9\%) areas prior to death. If decedents missing data on the timing of death were also more likely to have been classified as SCD had the death been witnessed and the timing of death were known, then the reported RR estimates for the association between lower nINC and SCD are biased downward and toward the null. The results of our sensitivity analysis, which incorporated weights for each race-gender group based upon the predicted probability of having an unknown timing of death, indicated bias in the hypothesized direction, although the findings did not change the interpretation of our results (data not shown).

A limitation of these data is that relatively few deaths occurred among persons of other race/ ethnicities during the study period; thus, we were not able to include mortality estimates for race/ethnicities other than black or white. Further, individual-level variables, whether related to medical history or SES, are not typically available in surveillance studies, and do not exist for the current study. While neighborhood and individual measures of SES are modestly correlated (27), neighborhood socioeconomic effects on morbidity and mortality tend to be robust in the presence of individual-level SES $(27,28)$. Consistent with our previous work, the use of a single-variable nSES measure produced results of similar magnitude and precision when compared to a more complex composite index measure of nSES (23). In addition, we found similar results regardless of which single-variable nSES measure was used. The use of a single-variable nSES measure may be appealing for its relative simplicity of use in a surveillance setting.

The influence of nSES, independent of individual-level SES, on out-of-hospital fatal CHD and SCD could be due to health care access, presence or absence of environmental stressors, and level of social support among neighborhood residents. Considering that the observed associations were greater in magnitude for women compared to men, it may be that the mechanisms referred to, above, play a larger role among women. However, these data do not capture the underlying factors which may explain these findings. Measures of the neighborhood context remain useful tools for population-based surveillance efforts to document and monitor socioeconomic disparities in mortality over time. 


\section{Supplementary Material}

Refer to Web version on PubMed Central for supplementary material.

\section{Acknowledgments}

The Atherosclerosis Risk in Communities Study is carried out as a collaborative study supported by National Heart, Lung, and Blood Institute contracts N01-HC-55015, N01-HC-55016, N01-HC-55018, N01-HC-55019, N01-

HC-55020, N01-HC-55021, and N01-HC-55022. The authors thank the staff of the ARIC study for their important contributions.

\section{ABBREVIATIONS}

$\begin{array}{ll}\text { ARIC } & \text { Atherosclerosis Risk in Communities study } \\ \text { CHD } & \text { coronary heart disease } \\ \text { CI } & \text { confidence interval } \\ \text { CT } & \text { census tract } \\ \text { H } & \text { high } \\ \text { ICD } & \text { international classification of diseases } \\ \text { L } & \text { low } \\ \text { M } & \text { medium } \\ \text { MD } & \text { Maryland } \\ \text { MN } & \text { Minnesota } \\ \text { MS } & \text { Mississippi } \\ \text { NC } & \text { North Carolina } \\ \text { nINC } & \text { neighborhood median household income } \\ \text { nSES } & \text { neighborhood socioeconomic status } \\ \text { RR } & \text { rate ratio } \\ \text { SCD } & \text { sudden cardiac death } \\ \text { SMR } & \text { standardized mortality ratio } \\ \text { US } & \text { United States }\end{array}$

\section{REFERENCES}

1. Chambless L, Keil U, Dobson A, et al. Population versus clinical view of case fatality from acute coronary heart disease: Results from the WHO MONICA Project 1985-1990. Circulation. 1997; 96(11):3849-59. [PubMed: 9403607]

2. De Henauw S, De Bacquer D, de Smet P, et al. Trends in coronary heart disease in two Belgian areas: results from the MONICA Ghent-Charleroi Study. J Epidemiol Community Health. 1999; 53(2):89-98. [PubMed: 10396469]

3. Saito I, Folsom AR, Aono H, et al. Comparison of fatal coronary heart disease occurrence based on population surveys in Japan and the USA. Int J Epidemiol. 2000; 29(5):837-44. [PubMed: 11034966]

4. Marmot MG, Shipley MJ, Hemingway H, et al. Biological and behavioural explanations of social inequalities in coronary heart disease: the Whitehall II Study. Diabetologia. 2008; 51(11):1980-8. [PubMed: 18777168] 
5. Kostis JB, Wilson AC, Lacy CR, et al. Time trends in the occurrence and outcome of acute myocardial infarction and coronary heart disease death between 1986 and 1996 (a New Jersey statewide study). Am J Cardiol. 2001; 88(8):651-2. [PubMed: 11564389]

6. Rosamond WD, Chambless LE, Folsom AR, et al. Trends in the incidence of myocardial infarction and in mortality due to coronary heart disease, 1987 to 1994. N Engl J Med. 1998; 339(13):861-7. [PubMed: 9744969]

7. Tunstall-Pedoe H, Kuulasmaa K, Mahonen M, et al. Contribution of trends in survival and coronaryevent rates to changes in coronary heart disease mortality: 10-year results from 37 WHO MONICA project populations. Monitoring trends and determinants in cardiovascular disease. Lancet. 1999; 353(9164):1547-57. [PubMed: 10334252]

8. Saito I, Ozawa H, Aono H, et al. Trends in fatal coronary heart disease among people aged 25-74 years in Oita City, Japan, from 1987-1998. Journal of Clinical Epidemiology. 2002; 55(5):469-76. [PubMed: 12007550]

9. Capewell S, MacIntyre K, Stewart S, et al. Age, sex, and social trends in out-of-hospital cardiac deaths in Scotland 1986-95: a retrospective cohort study. The Lancet. 2001; 358(9289):1213-7.

10. Hemingway H, Malik M, Marmot M. Social and psychosocial influences on sudden cardiac death, ventricular arrhythmia and cardiac autonomic function. Eur Heart J. 2001; 22(13):1082-101. [PubMed: 11428849]

11. Galea S, Blaney S, Nandi A, et al. Explaining racial disparities in incidence of and survival from out-of-hospital cardiac arrest. Am J Epidemiol. 2007; 166(5):534-43. [PubMed: 17584756]

12. Ni H, Coady S, Rosamond W, et al. Trends from 1987 to 2004 in sudden death due to coronary heart disease: the Atherosclerosis Risk in Communities (ARIC) study. Am Heart J. 2009; 157(1): 46-52. [PubMed: 19081395]

13. Nichol G, Thomas E, Callaway CW. Regional variation in out-of-hospital cardiac arrest incidence and outcome. Jama. 2008; 300(12):1423-31. [PubMed: 18812533]

14. Gillum RF. Geographic variation in sudden coronary death. Am Heart J. 1990; 119(2 pt 1):380-9. [PubMed: 2301226]

15. White AD, Folsom AR, Chambless LE, et al. Community surveillance of coronary heart disease in the Atherosclerosis Risk in Communities (ARIC) Study: methods and initial two years' experience. J Clin Epidemiol. 1996; 49(2):223-33. [PubMed: 8606324]

16. ARIC. Surveillance Component Procedures. Atherosclerosis Risk in Communities Study. 2009.

17. Whitsel EA, Rose KM, Wood JL, et al. Accuracy and repeatability of commercial geocoding. Am J Epidemiol. 2004; 160(10):1023-9. [PubMed: 15522859]

18. Diez-Roux AV, Merkin SS, Arnett D, et al. Neighborhood of residence and incidence of coronary heart disease. N Engl J Med. 2001; 345(2):99-106. [PubMed: 11450679]

19. Borrell LN, Diez Roux AV, Rose K, et al. Neighbourhood characteristics and mortality in the Atherosclerosis Risk in Communities Study. Int J Epidemiol. 2004; 33(2):398-407. [PubMed: 15082648]

20. Diez-Roux AV, Kiefe CI, Jacobs DR, et al. Area characteristics and individual-level socioeconomic position indicators in three population-based epidemiologic studies. Annals of Epidemiology. 2001; 11(6):395-405. [PubMed: 11454499]

21. Krieger N, Waterman PD, Chen JT, et al. Monitoring socioeconomic inequalities in sexually transmitted infections, tuberculosis, and violence: geocoding and choice of area-based socioeconomic measures--the public health disparities geocoding project (US). Public Health Rep. 2003; 118(3):240-60. [PubMed: 12766219]

22. Census UBot. Geographical areas reference manual. 2002. http://www.census.gov/geo/www/garm.html). (Accessed)

23. Rose KM, Suchindran CM, Foraker RE, et al. Neighborhood Disparities in Incident Hospitalized Myocardial Infarction in Four U.S. Communities: The ARIC Surveillance Study. Annals of Epidemiology. 2009; 19(12):867-74. [PubMed: 19815428]

24. Krieger N, Chen JT, Waterman PD, et al. Race/ethnicity, gender, and monitoring socioeconomic gradients in health: a comparison of area-based socioeconomic measures--the public health disparities geocoding project. Am J Public Health. 2003; 93(10):1655-71. [PubMed: 14534218] 
25. Wagstaff A, Watanabe N. What difference does the choice of SES make in health inequality measurement? Health Economics. 2003; 12(10):885-90. [PubMed: 14508873]

26. Shavers VL. Measurement of socioeconomic status in health disparities research. J Natl Med Assoc. 2007; 99(9):1013-23. [PubMed: 17913111]

27. Diez Roux AV, Kiefe CI, Jacobs J,DR, et al. Area characteristics and individual-level socioeconomic position indicators in three population-based epidemiologic studies. Annals of Epidemiology. 2001; 11(6):395-405. [PubMed: 11454499]

28. Krieger N. Overcoming the absence of socioeconomic data in medical records: validation and application of a census-based methodology. American Journal of Public Health. 1992; 82(5) 


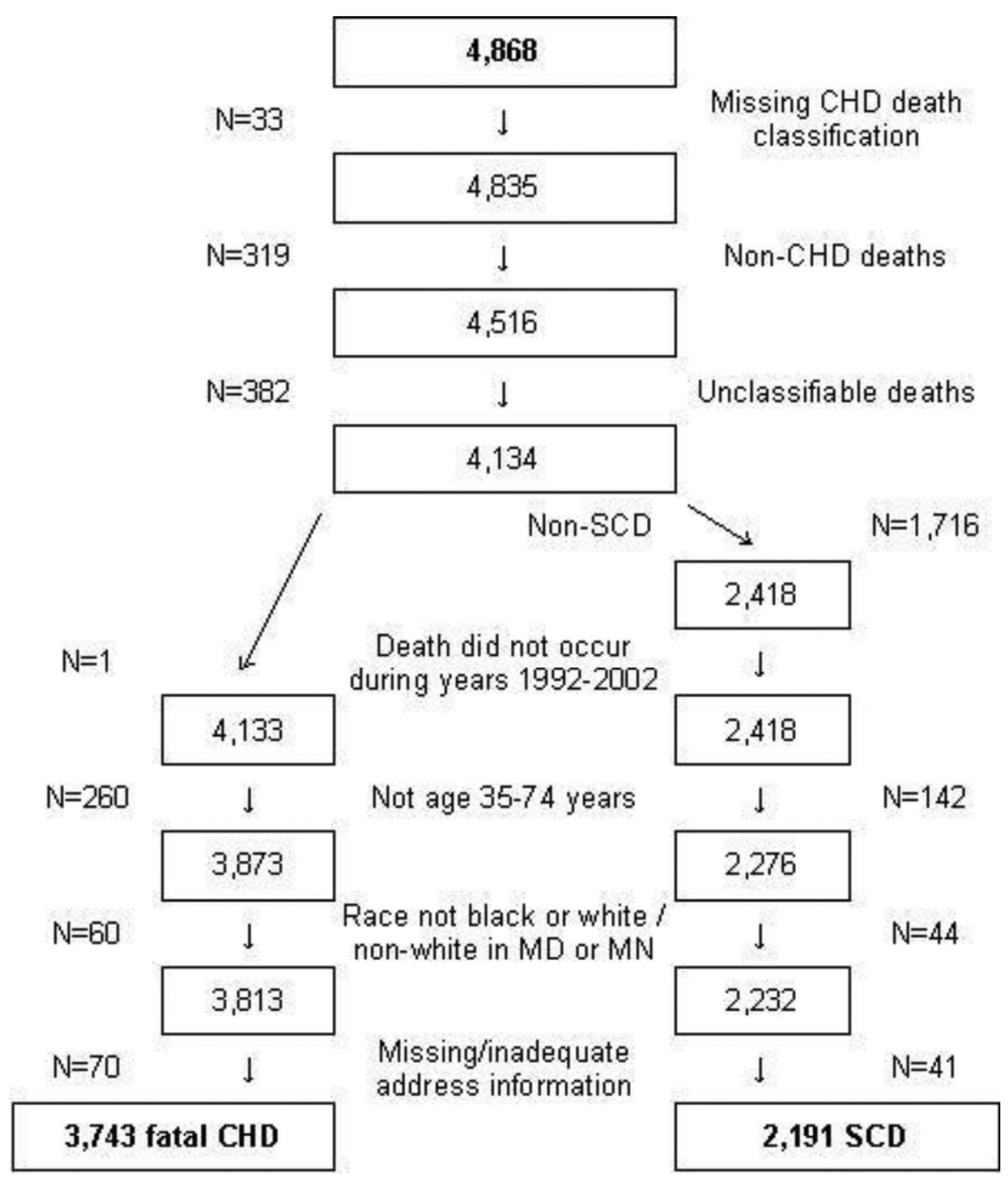

Figure 1.

Exclusions for the Study of Out-of-hospital Deaths in ARIC Community Surveillance (1992-2002) 
By Race-Gender Group
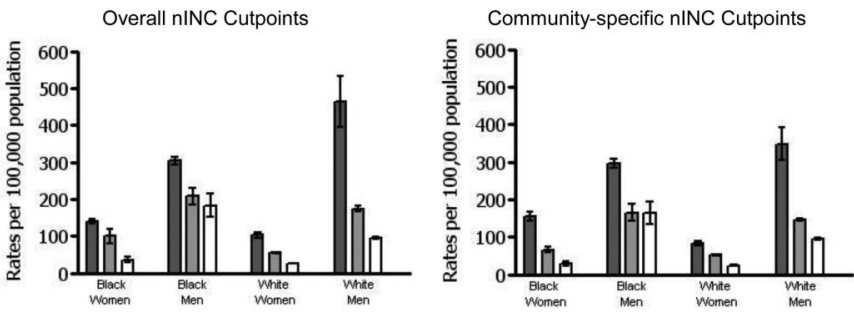

By Study Community
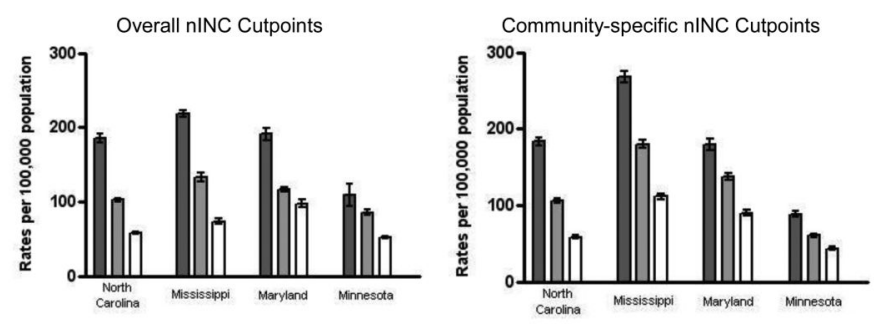

Figure 2.

(a-d). Age-adjusted Out-of-hospital CHD Death Rates by Median Household Income (nINC), by Race-Gender Group and Study Community: ARIC Community Surveillance (1992-2002) 


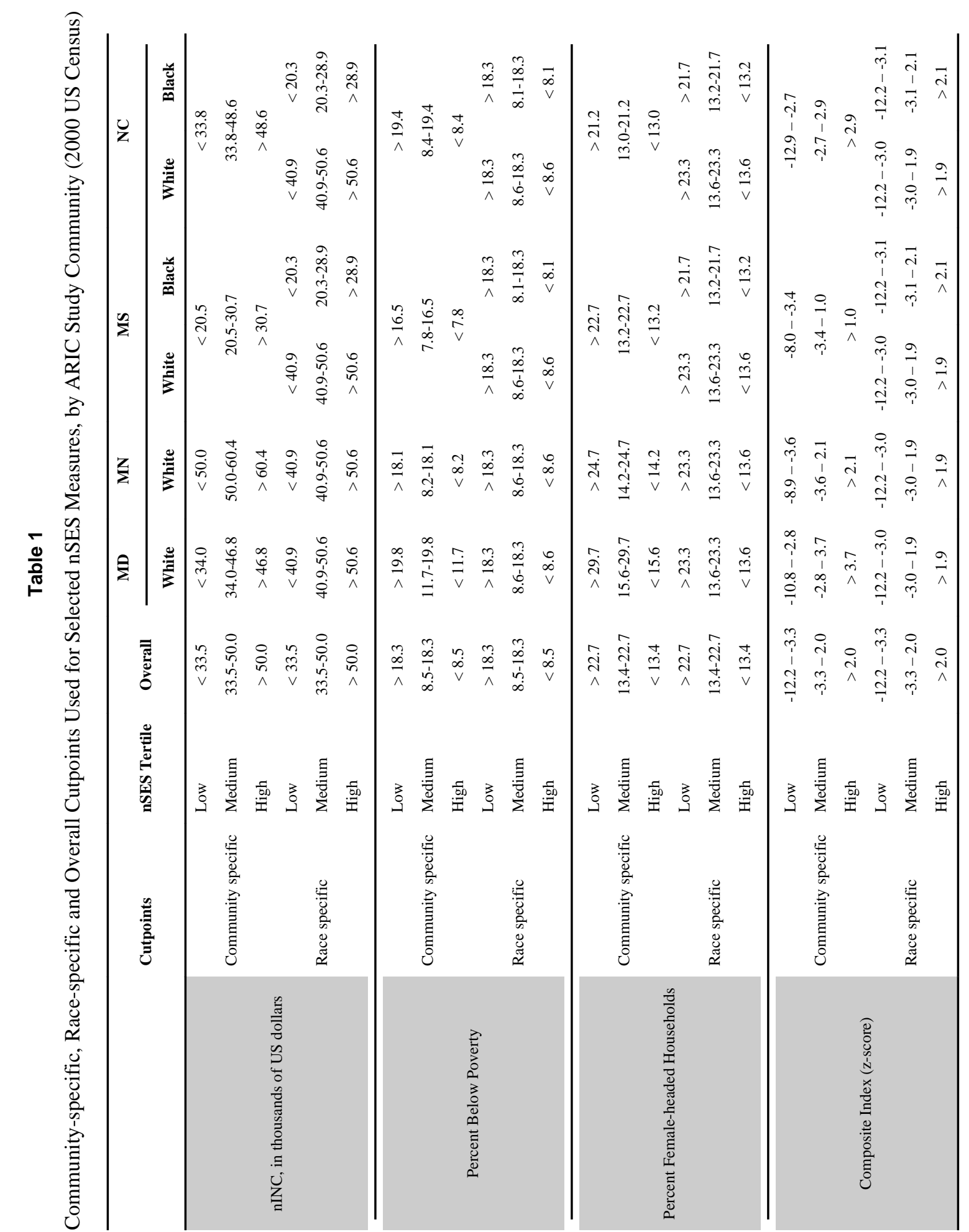


Table 2

Characteristics (N) of Eligible Population by ARIC Study Community, 2000 Census

\begin{tabular}{|c|c|c|c|c|}
\hline & Washington Co. Maryland & Minneapolis Minnesota & Jackson (city) Mississippi & $\begin{array}{c}\text { Forsyth Co. North } \\
\text { Carolina }\end{array}$ \\
\hline \multicolumn{5}{|l|}{ Race-gender group } \\
\hline Black Women & 1,330 & 4,694 & 26,976 & 18,181 \\
\hline Black Men & 1,220 & 4,380 & 21,545 & 15,175 \\
\hline White Women & 29,048 & 48,329 & 8,491 & 53,272 \\
\hline White Men & 27,033 & 45,168 & 7,137 & 47,887 \\
\hline Total population $*$ & 58,631 & 102,571 & 64,149 & 134,515 \\
\hline Census tracts & 31 & 55 & 43 & 75 \\
\hline $\begin{array}{l}\text { Average persons per census } \\
\text { tract }\end{array}$ & 1,891 & 1,865 & 1,492 & 1,794 \\
\hline
\end{tabular}


Table 3

Association of Low, Medium and High nSES (RR, 95\% CI) with the Incidence of Out-of-hospital CHD Death*: ARIC Community Surveillance (1992-2002)

\begin{tabular}{|c|c|c|c|c|}
\hline & Black Women & Black Men & White Women & White Men \\
\hline \multicolumn{5}{|c|}{ nINC (Overall) } \\
\hline Low & $2.96(1.65,5.29)$ & $1.68(1.14,2.47)$ & $2.89(2.29,3.65)$ & $2.32(1.92,2.81)$ \\
\hline Medium & $1.61(0.85,3.05)$ & $1.18(0.77,1.82)$ & $1.70(1.40,2.07)$ & $1.59(1.36,1.85)$ \\
\hline High & 1.00 & 1.00 & 1.00 & 1.00 \\
\hline \multicolumn{5}{|c|}{ nINC (Community-specific) } \\
\hline Low & $2.42(1.67,3.49)$ & $1.56(1.18,2.05)$ & $2.54(2.05,3.16)$ & $2.20(1.86,2.60)$ \\
\hline Medium & $1.42(0.97,2.09)$ & $1.09(0.81,1.45)$ & $1.81(1.50,2.18)$ & $1.63(1.42,1.89)$ \\
\hline High & 1.00 & 1.00 & 1.00 & 1.00 \\
\hline \multicolumn{5}{|c|}{ nINC (Race-specific) } \\
\hline Low & $3.00(1.90,4.74)$ & $1.62(1.09,2.40)$ & $2.59(2.09,3.22)$ & $2.11(1.79,2.50)$ \\
\hline Medium & $2.48(1.46,4.19)$ & $1.07(0.63,1.83)$ & $1.72(1.38,2.15)$ & $1.45(1.22,1.72)$ \\
\hline High & 1.00 & 1.00 & 1.00 & 1.00 \\
\hline \multicolumn{5}{|c|}{ Percent Individuals Below } \\
\hline \multicolumn{5}{|l|}{ Poverty } \\
\hline Low & $2.21(1.08,4.51)$ & $1.39(0.88,2.18)$ & $2.62(2.07,3.33)$ & $2.55(2.12,3.06)$ \\
\hline Medium & $1.04(0.48,2.25)$ & $0.81(0.50,1.34)$ & $1.50(1.23,1.82)$ & $1.54(1.33,1.78)$ \\
\hline High & 1.00 & 1.00 & 1.00 & 1.00 \\
\hline \multicolumn{5}{|c|}{ Percent Female-headed } \\
\hline \multicolumn{5}{|l|}{ Households } \\
\hline Low & $2.25(1.13,4.48)$ & $1.27(0.84,1.92)$ & $2.44(1.95,3.04)$ & $2.02(1.68,2.44)$ \\
\hline Medium & $1.60(0.77,3.33)$ & $0.84(0.53,1.32)$ & $1.60(1.32,1.95)$ & $1.36(1.17,1.60)$ \\
\hline High & 1.00 & 1.00 & 1.00 & 1.00 \\
\hline \multicolumn{5}{|c|}{ Percent College-educated } \\
\hline Low & $1.92(1.38,2.67)$ & $1.57(1.22,2.03)$ & $2.56(1.99,3.30)$ & $2.33(1.89,2.86)$ \\
\hline Medium & $1.28(0.91,1.81)$ & $1.12(0.86,1.46)$ & $1.86(1.54,2.26)$ & $1.53(1.32,1.77)$ \\
\hline High & 1.00 & 1.00 & 1.00 & 1.00 \\
\hline \multicolumn{5}{|c|}{ Percent High School-educated } \\
\hline Low & $2.42(1.61,3.64)$ & $1.83(1.35,2.49)$ & $2.50(1.98,3.16)$ & $2.38(1.98,2.85)$ \\
\hline Medium & $1.42(0.89,2.28)$ & $1.48(1.04,2.10)$ & $1.73(1.41,2.12)$ & $1.51(1.29,1.77)$ \\
\hline High & 1.00 & 1.00 & 1.00 & 1.00 \\
\hline \multicolumn{5}{|c|}{ Composite Index } \\
\hline Low & $2.77(1.65,4.64)$ & $1.61(1.12,2.31)$ & $3.27(2.62,4.08)$ & $2.66(2.20,3.22)$ \\
\hline Medium & $1.39(0.78,2.47)$ & $1.03(0.69,1.54)$ & $1.98(1.66,2.36)$ & $1.44(1.25,1.67)$ \\
\hline High & 1.00 & 1.00 & 1.00 & 1.00 \\
\hline
\end{tabular}


Table 4

Association of Low, Medium and High nSES (RR, 95\% CI) with the Incidence of SCD*: ARIC Community Surveillance (1992-2002)

\begin{tabular}{|c|c|c|c|c|}
\hline & Black Women & Black Men & White Women & White Men \\
\hline \multicolumn{5}{|c|}{ nINC (Overall) } \\
\hline Low & $4.36(1.72,11.0)$ & $2.01(1.24,3.28)$ & $3.11(2.29,4.21)$ & $2.17(1.72,2.75)$ \\
\hline Medium & $2.25(0.84,6.05)$ & $1.29(0.75,2.21)$ & $1.58(1.22,2.06)$ & $1.55(1.28,1.87)$ \\
\hline High & 1.00 & 1.00 & 1.00 & 1.00 \\
\hline \multicolumn{5}{|c|}{ nINC (Community-specific) } \\
\hline Low & $2.60(1.53,4.42)$ & $1.57(1.11,2.23)$ & $2.61(1.94,3.50)$ & $2.00(1.62,2.46)$ \\
\hline Medium & $1.47(0.84,2.57)$ & $1.11(0.77,1.61)$ & $1.87(1.45,2.41)$ & $1.63(1.36,1.94)$ \\
\hline High & 1.00 & 1.00 & 1.00 & 1.00 \\
\hline \multicolumn{5}{|c|}{ nINC (Race-specific) } \\
\hline Low & $3.94(2.05,7.58)$ & $1.93(1.16,3.20)$ & $2.61(1.96,3.49)$ & $2.00(1.63,2.45)$ \\
\hline Medium & $2.19(1.02,4.73)$ & $1.35(0.70,2.61)$ & $1.56(1.15,2.11)$ & $1.37(1.11,1.69)$ \\
\hline High & 1.00 & 1.00 & 1.00 & 1.00 \\
\hline \multicolumn{5}{|c|}{ Percent Individuals Below } \\
\hline \multicolumn{5}{|l|}{ Poverty } \\
\hline Low & $2.28(0.92,5.66)$ & $1.17(0.71,1.93)$ & $3.15(2.32,4.28)$ & $2.42(1.93,3.03)$ \\
\hline Medium & $0.85(0.31,2.31)$ & $0.53(0.30,0.94)$ & $1.75(1.35,2.26)$ & $1.61(1.35,1.92)$ \\
\hline High & 1.00 & 1.00 & 1.00 & 1.00 \\
\hline \multicolumn{5}{|c|}{ Percent Female-headed } \\
\hline \multicolumn{5}{|l|}{ Households } \\
\hline Low & $2.13(0.90,5.01)$ & $1.15(0.72,1.85)$ & $2.55(1.92,3.40)$ & $1.92(1.53,2.40)$ \\
\hline Medium & $1.46(0.58,3.66)$ & $0.71(0.41,1.20)$ & $1.42(1.10,1.85)$ & $1.37(1.13,1.65)$ \\
\hline High & 1.00 & 1.00 & 1.00 & 1.00 \\
\hline \multicolumn{5}{|c|}{ Percent College-educated } \\
\hline Low & $1.81(1.14,2.89)$ & $1.82(1.32,2.52)$ & $2.99(2.17,4.12)$ & $2.41(1.89,3.06)$ \\
\hline Medium & $1.24(0.76,2.01)$ & $1.32(0.94,1.84)$ & $1.88(1.46,2.43)$ & $1.48(1.24,1.77)$ \\
\hline High & 1.00 & 1.00 & 1.00 & 1.00 \\
\hline \multicolumn{5}{|c|}{ Percent High School-educated } \\
\hline Low & $3.33(1.75,6.33)$ & $2.14(1.45,3.17)$ & $2.63(1.93,3.58)$ & $2.33(1.87,2.90)$ \\
\hline Medium & $2.36(1.16,4.79)$ & $1.68(1.08,2.61)$ & $1.71(1.31,2.25)$ & $1.37(1.12,1.66)$ \\
\hline High & 1.00 & 1.00 & 1.00 & 1.00 \\
\hline \multicolumn{5}{|c|}{ Composite Index } \\
\hline Low & $5.35(2.16,13.3)$ & $2.06(1.31,3.25)$ & $3.64(2.69,4.94)$ & $2.66(2.11,3.35)$ \\
\hline Medium & $2.53(0.97,6.64)$ & $1.14(0.69,1.90)$ & $2.12(1.66,2.70)$ & $1.37(1.15,1.63)$ \\
\hline High & 1.00 & 1.00 & 1.00 & 1.00 \\
\hline
\end{tabular}

